\title{
Revista Colombiana de

\section{Uso de los sistemas de presión negativa en el tratamiento de infecciones asociadas a dispositivos: "una vieja terapia con un uso novedoso"'}

\author{
William Fernando Bautista Vargas ${ }^{a, *}$, Diego Andrés Rodriguez Guerrero ${ }^{a}$ \\ y Luis Carlos Sáenz ${ }^{\mathrm{b}}$
}

a Departamento de Electrofisiología, Universidad de La Sabana, Bogotá, Colombia

b Departamento de Electrofisiología, Fundación Cardioinfantil, Bogotá, Colombia

Recibido el 2 de agosto de 2015; aceptado el 21 de enero de 2016

Disponible en Internet el 19 de marzo de 2016

\section{PALABRAS CLAVE \\ Dispositivo; \\ Endocarditis; \\ Cables marcapasos}

\begin{abstract}
Resumen
Introducción: El cuidado de los pacientes con infecciones asociadas al dispositivo sin endocarditis, incluye: la extracción completa y la realización de capsulectomía para evitar la reinfección. El uso de nuevos elementos como el sistema de la presión negativa es una terapia novedosa que ha demostrado disminuir: los tiempos de recuperación, el tiempo de estancia intrahospitalaria, la necesidad de nuevas intervenciones por sangrado o lavado y el tiempo de espera para un nuevo implante. Nuestro objetivo es describir la experiencia de un centro de cuarto nivel, en el manejo de las infecciones asociadas a los dispositivos sin endocarditis, con el uso de la terapia de presión negativa como parte del manejo.

Metodología: Se realizó una descripción retrospectiva de la experiencia en el manejo de la terapia de presión negativa en pacientes con infecciones asociadas a dispositivo, en un centro de cuarto nivel. Se exponen cinco casos, a los que se les realizó: la extracción completa del dispositivo, la capsulectomía anterior y posterior, el lavado quirúrgico y el implante de la terapia con presión negativa.

Conclusión: La experiencia presentada demuestra que el uso de la terapia de presión negativa es un protocolo de tratamiento novedoso, que en nuestros pacientes con infección asociada a dispositivo sin endocarditis, es segura y de fácil utilización.

(c) 2016 Sociedad Colombiana de Cardiología y Cirugía Cardiovascular. Publicado por Elsevier España, S.L.U. Este es un artículo Open Access bajo la licencia CC BY-NC-ND (http:// creativecommons.org/licenses/by-nc-nd/4.0/).
\end{abstract}

\footnotetext{
* Autor para correspondencia.

Correos electrónicos: williambautista679@gmail.com, williambautista679@hotmail.com (W.F. Bautista Vargas).
} 


\section{KEYWORDS}

Device;

Endocarditis;

Pacemaker wires
Use of negative pressure systems for the management of infections associated to devices: "an old therapy with a new use"

\begin{abstract}
Introduction: Care for patients with infections associated to the device without endocarditis include complete extraction and performing a capsulectomy to avoid reinfection. The use of new elements, such as the negative pressure system, is a new therapy that has shown to reduce recovery time, inpatient stay, need of new interventions due to bleeding or washing and waiting time for a new implant. The goal is to describe the experience of a fourth level centre for managing infections associated with devices without endocarditis using a negative pressure system as part of the therapy.

Methods: Descriptive retrospective study of the experience of negative pressure therapy for managing patients with device-related infections at a fourth level centre. Five cases are exposed where complete removal of the device, anterior and posterior capsulectomy, surgical washing and negative pressure therapy were performed.

Conclusion: Presented experience shows that the use of negative pressure therapy is a new therapy protocol that is safe and easy for managing our patients with an infection associated to the device without endocarditis.

(C) 2016 Sociedad Colombiana de Cardiología y Cirugía Cardiovascular. Published by Elsevier España, S.L.U. This is an open access article under the CC BY-NC-ND license (http:// creativecommons.org/licenses/by-nc-nd/4.0/).
\end{abstract}

\section{Introducción}

El paciente con infección asociada a dispositivo se considera de alto riesgo de complicacióny de muerte. Ante esta infección se debe evaluar si existe: compromiso sistémico, signos de respuesta inflamatoria, presencia de abscesos, endocarditis o disfunción multiorgánica; condiciones que ponen en riesgo la vida de los pacientes ${ }^{1}$. La primera valoración debe hacerse por los médicos entrenados en manejo de infección de dispositivos, de tal forma que sean ellos quienes dirijan las intervenciones diagnósticas y terapéuticas a realizar ${ }^{1}$. Queremos hacer énfasis en el manejo de los pacientes con infección asociada a dispositivo sin endocarditis, definida como la presencia de cambios inflamatorios en el bolsillo (eritema, calor, rubor, dolor), y respuesta inflamatoria sistémica activa pero sin criterios de endocarditis ${ }^{2}$.

Una antigua práctica, tiene una nueva indicación en la electrofisiología. El uso de los sistemas de presión negativa (SPN), tiene varios reportes de éxito en el manejo de las infecciones asociadas al dispositivo sin endocarditis $(\text { IADSE) })^{3}$. El SPN tiene un funcionamiento complejo: está basado en las propiedades físicas del contacto de una espuma y el tejido ${ }^{4}$.

Esta terapia se ha evaluado de forma exitosa en múltiples escenarios clínicos, como: intervenciones en heridas abdominales y ortopédicas ${ }^{3,4}$.

Para la utilización del sistema, se debe introducir una espuma en el bolsillo vacío, posteriormente, un aislante fijado a la piel hace que el sistema quede cerrado; se conecta un tubo a la interfase espuma-tejido y a un sistema de succión que genera la presión negativa (Fig. 1). El material que se pone en contacto con la herida puede ser de dos tipos: éter de poliuretano (espuma negra) o polivinil alcohol (espuma blanca) $)^{3,4}$.

\section{Materiales y métodos}

Se presenta la experiencia de un hospital de cuarto nivel, en el uso de los SNP para el manejo de las IADSE. Durante el año 2015, los autores adoptamos el uso de los SPN en los pacientes con las IADSE, con la intención de disminuir los días de estancia hospitalaria y la necesidad de reintervenciones por sangrados. Esta se introdujo como complemento al protocolo previo que incluye: la extracción del dispositivo y los electrodos, la capsulectomía anterior, la posterior y el lavado de bolsillo con solución salina.

Todos los pacientes incluidos recibieron el mismo tratamiento, se realizó: el explante del dispositivo y la extracción manual. Se les implantó la espuma de éter de poliuretano (Granufoam ${ }^{\circledR}$ ), con sello de la cavidad, se inició la terapia con el sistema de presión negativa continúa a $125 \mathrm{mmHg}$ por 72 a 96 horas. Si el paciente se encontraba anticoagulado, se utilizó la presión negativa de $75 \mathrm{mmHg}$. La terapia se usó en promedio por 5 días y se obtuvieron en promedio drenajes de $18 \mathrm{cc} /$ día. El retiro de la terapia se realizaba cuando el drenaje era claro, tenía secreción escasa y existía mejoría clínica. Adicionalmente, se debió retirar la terapia, si empeoraba la condición clínica o el paciente tenía indicación de otro tipo de tratamiento como: reintervención quirúrgica, cirugía de extracción abierta u otra conducta.

\section{Resultados}

Esta serie cuenta con cinco casos (cuatro hombres y una mujer, con edad mínima de 32 años y máxima de 96 años), todos con las IADSE (tabla 1).

A continuación se hará un reporte de lo más relevante de los pacientes:

Paciente 1: hombre de 39 años de edad, portador de válvula mecánica en posición aórtica (Fig. 2). Dada la 


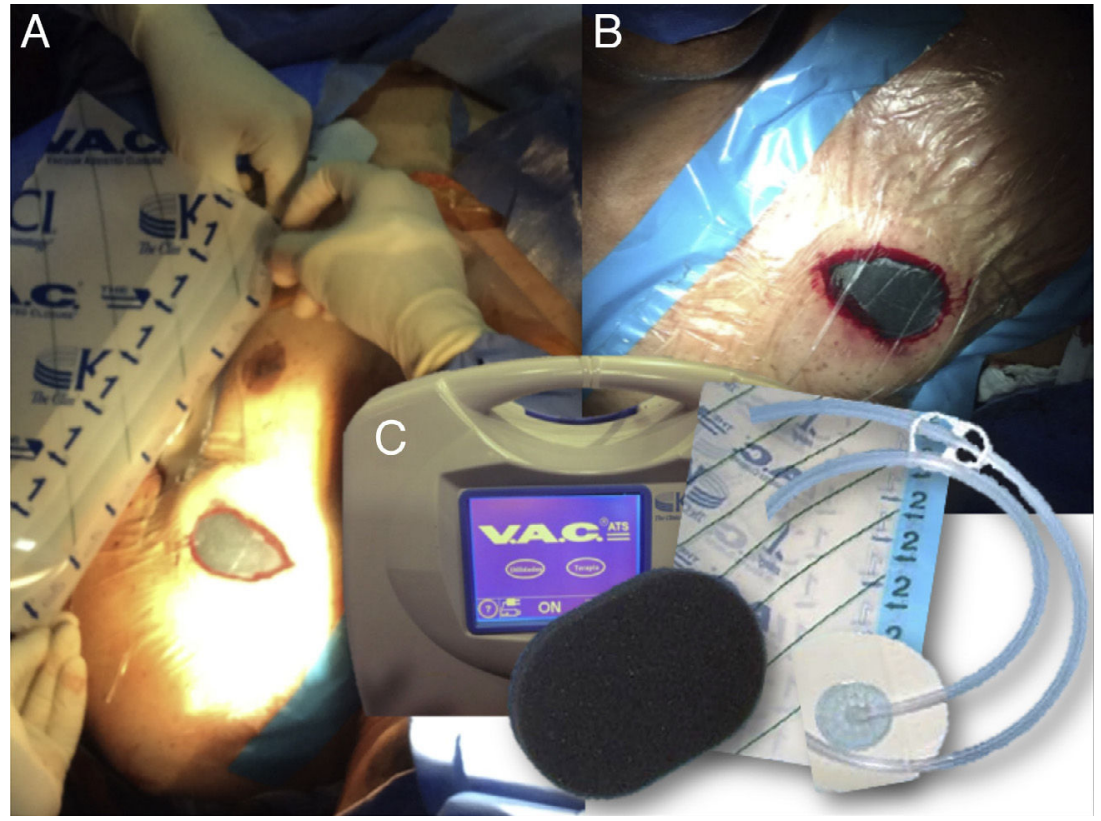

Figura 1 A. Sistema de presión negativa; Implante de membrana y espuma.

B. Cavidad herméticamente sellada con espuma y membrana.

C. Sistema de presión negativa con sus componentes, espuma, succión, membrana y conector.

evidencia de las IADSE, se le realizó explante de dispositivo (explante quirúrgico, más tracción manual de los electrodos) y resección de tumoración pectoral, la cual no mostró malignidad. Este paciente recibió tratamiento con los SPN por 6 días, durante este tiempo estuvo anticoagulado con heparina sódica en infusión continúa. A pesar de que no se realizó aislamiento microbiológico en ninguna muestra, el paciente recibió antibioticoterapia en esquema de 21 días e implante posterior de marcapasos, sin presentar complicaciones relacionadas con el sangrado o la reintervención. La estancia hospitalaria fue de 28 días.

Paciente 2: mujer de 32 años de edad, con infección asociada a marcapasos, a la que se le realizó extracción en dos tiempos: en un primer tiempo se le realizó extracción percutánea del dispositivo y tracción manual de los electrodos con fractura del electrodo auricular. Se inició la

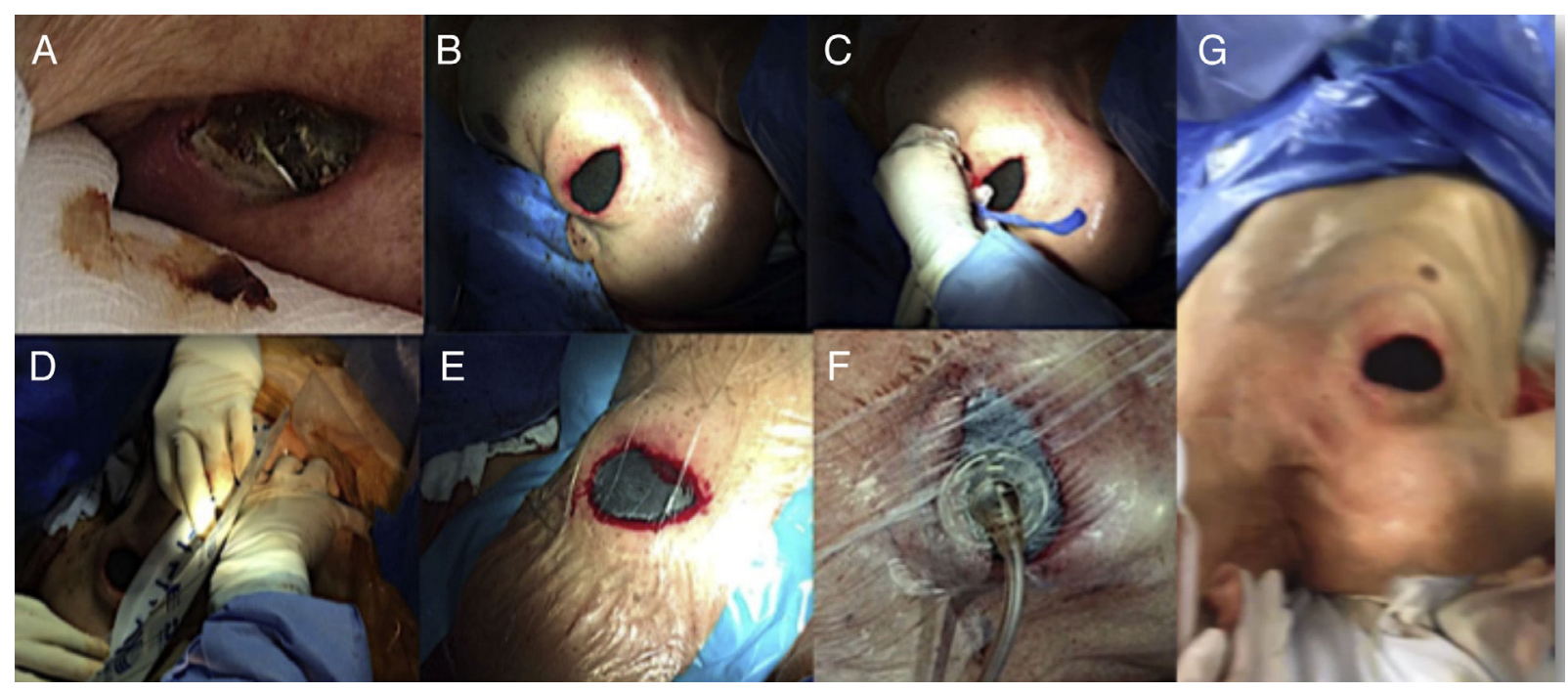

Figura 2 Paciente de 96 años con exposición del dispositivo, a quien se le implantó sistema de presión negativa.

A. Imagen inicial con extrusión de dispositivo.

B. Implante de espuma.

C-D. Implante de membrana.

E-F. Implante de succión negativa.

G. Imagen de posición de abordaje para implante de sistema de presión negativa. 
Tabla 1 Características de los pacientes tratados con terapia de presión negativa en enero y julio de $2015(n=5)$

\begin{tabular}{lcl}
\hline & Mediana o n & Rango o \% \\
\hline Edad (años) & 40 & $(28-96)$ \\
Sexo masculino & 4 & 80 \\
Estancia hospitalaria (días) & 24 & $(9-28)$ \\
Días de tratamiento con & 6 & $(2-9)$ \\
$\quad$ presión negativa (días) & & \\
Drenaje (cc) & 11 & $(5-35)$ \\
Aislamiento microbiológico & 2 & $40 \%$ \\
\hline
\end{tabular}

antibioticoterapia y la terapia de presión negativa. Dada la persistencia de los síntomas, se requirió abordaje quirúrgico por esternotomía para retiro de punta del electrodo fracturado. En el seguimiento no presentó aislamiento microbiológico en la sangre ni en material explantado.

La paciente no requirió nuevo implante, dado la ausencia de síntomas de disfunción sinusal, los cuales habían sido la indicación inicial de implante del dispositivo.

Paciente 3: hombre de 39 años de edad, con disfunción ventricular, cardiopatía dilatada deorigen chagásico, en quien se le realizó implante por prevención primaria de muerte súbita, requirió la extracción y uso de los SPN por 2 días, con posterior implante del dispositivo contralateral. -Pacientes con aislamiento microbiológico:

Paciente 4: hombre de 62 años de edad, con aislamiento en la sangre de Staphylococcus aureus. Este paciente tenía infección asociada al implante del cardiodesfibrilador. Se usó la terapia de presión negativa durante 9 días y recibió antibioticoterapia con un esquema de 14 días. Se reimplantó una vez terminado el tratamiento antibiótico, con una estancia hospitalaria de 25 días.

Paciente 5: hombre de 96 años de edad, portador de marcapasos bicameral, por bloqueo auriculoventricular completo, a quien se le aisló en la sangre la Pseudomona Aureginosa. Este individuo requirió implante de electrodo transitorio y un nuevo implante de marcapasos definitivo a los 13 días del explante (Fig. 3). Este paciente duro con los SPN 6 días y su hospitalización fue de 24 días.

La evolución estética de la herida posterior al retiro de los SPN fue buena y aceptada por los pacientes, no existió cicatrización anormal en ninguno de los casos.

No existió mortalidad relacionada con el uso de los SPN, ni reinfección posterior a su retiro en el seguimiento a los 4 meses.

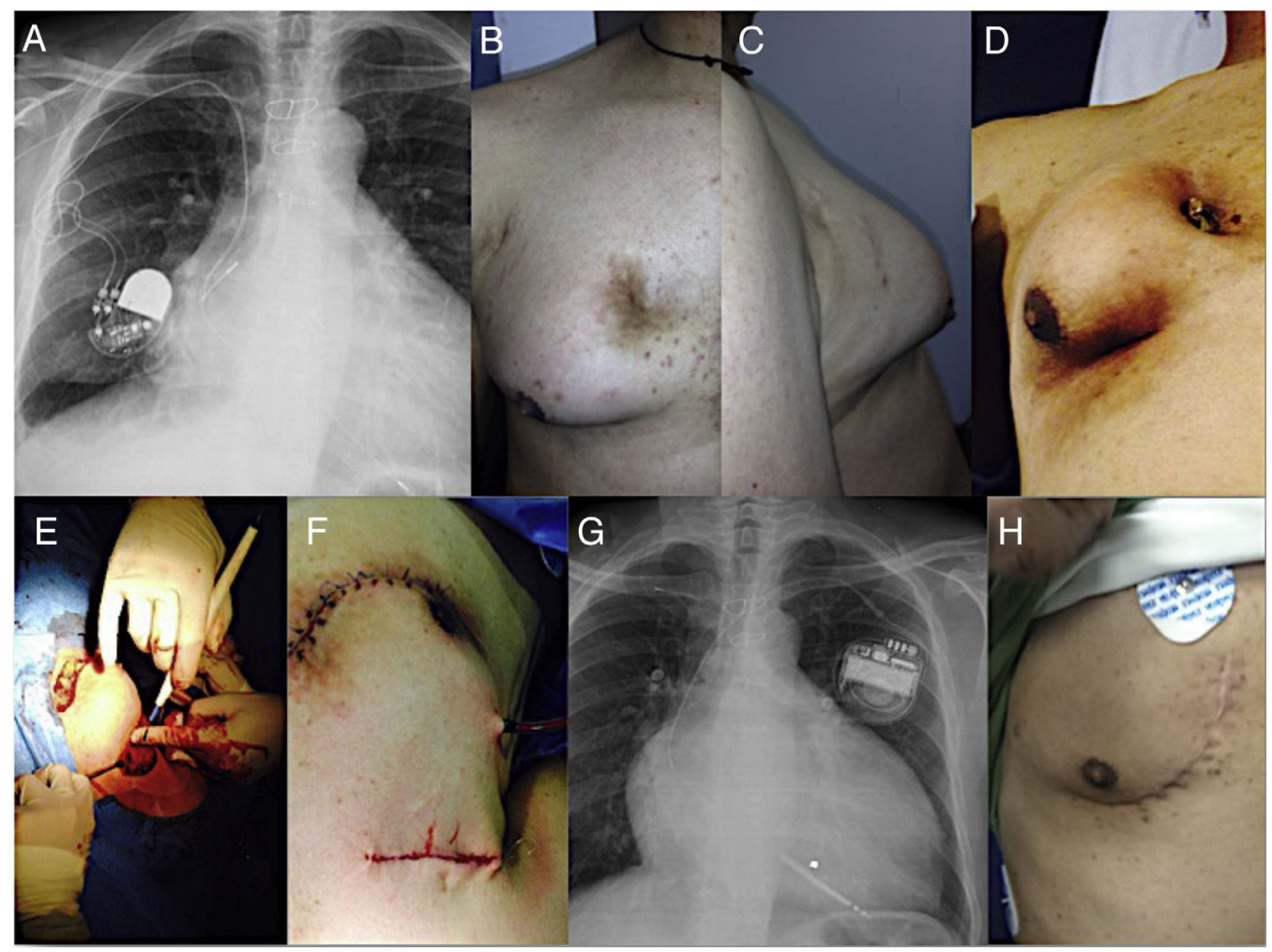

Figura 3 Paciente de 39 años con extrusión de dispositivo y ginecomastia.

A. Posición incial de generador.

B-C. Paciente 6 meses antes de presentar la extrusión del dispositivo.

D. Extrusión de dispositivo en pectral derecho.

E. Disección con explante de dispositivo.

F. Resección de tumoración asociada a generador extruido e implante de terapia con presión negativa.

G. Imagen con implante contralateral de generador.

H. Herida de extracción y resección de masa pectoral curada 2 meses después de la intervención. 


\section{Discusión}

Las indicaciones del explante en el manejo de la infección por los dispositivos de estimulación cardiaca incluyen: las IADSE, la infección asociada al generador cuando el riesgo de extracción de los cables no compromete la vida del paciente y la endocarditis asociada al dispositivo.

Este tipo de terapias requiere, además, antibioticoterapia especifica y dirigida a los gérmenes comunes ${ }^{3}$. En la serie de casos evaluada, los pacientes se llevaron al explante por infección asociada al generador y ninguno de ellos cumplía criterio de endocarditis asociada.

Reportamos el aislamiento microbiológico en 2 de 5 de los pacientes. En la literatura la prevalencia de la infección asociada a dispositivos llega hasta el 7\%, con una incidencia de 1/1000 dispositivos implantados, con una mortalidad que está entre el 5 y el $60 \%{ }^{3}$. En reportes del explante de dispositivos por infección; es más común el explante en pacientes portadores de marcapasos que en pacientes portadores de cardiodesfibriladores ${ }^{3,5}$. Hastael $78 \%$ de los aislamientos que se reportan son monobacterianos, $10 \%$ de los pacientes tienen crecimiento polibacteriano y hasta un $12 \%$ cultivos negativos $^{3}$. El $45 \%$ de los aislamientos son de Staphylococcus aureus, $44 \%$ de estos se reportan como meticilino-resistentes y solo del 9 al $18 \%$ son gérmenes gram negativos ${ }^{3,6}$. Dentro de los factores de riesgo reportados para la endocarditis asociada a dispositivo, se mencionan: la edad mayor a 69 años, los antecedentes de diabetes, la enfermedad reumática cardiaca, la insuficiencia renal, la endocarditis previa o el reimplante en la misma hospitalización 9 .

Dentro de las complicaciones reportadas, posteriormente, al explante por infección se encuentran: el requerimiento de la $\mathrm{UCl}$ en un $17 \%$, la falla renal aguda el $15 \%$, el hematoma el $3.4 \%$, el taponamiento cardiaco el $0.5 \%$ y la muerte intrahospitalaria en un $4.6 \%$.

El $57 \%$ de los pacientes con dispositivos explantados, requieren reimplante en la misma hospitalización y el $33 \%$ de los pacientes no requiere nuevo implante del dispositivo ${ }^{4,8,9}$.

En nuestra experiencia una paciente de cinco (20\%) no requirió nuevamente implante deldispositivo.

El uso de los SPN en infecciones no complicadas ha sido amplio, desde su lanzamiento como terapia para el manejo de infecciones, se estima que con diversas aplicaciones su uso oscila alrededor de 300 millones de usuarios en el mundo y se ha demostrado que es una terapia segura, aun en heridas no complicadas y en los pacientes con respuesta inflamatoria sistémica y sepsis ${ }^{5,7}$.

Los SPN como parte de un protocolo de manejo de las IADSE que incluya la extracción temprana de material infectado, resulta ser una terapia novedosa, segura y que acelera el proceso de curación de los pacientes, disminuye la necesidad de nuevas intervenciones lo cual es una ventaja sobre el manejo convencional de este tipo de infecciones ${ }^{4,9}$.

En una serie de pacientes, en donde se evaluó el uso de dispositivo de presión negativa para el manejo de infección asociada al generador, se encontró que la media de estancia hospitalaria fue de 34 días, con un caso de reinfección posterior a la terapia con presión negativa en el día 69 de seguimiento ${ }^{9}$. En nuestros pacientes, no existieron casos de reinfección posterior al retiro de la terapia de presión negativa, esto puede deberse a la intervención que proponemos como protocolo para las infecciones no complicadas que comprende: la extracción completa, la capsulectomía anterior y posterior, el lavado y el implante de la terapia de presión negativa.

La mediana de la estancia de nuestros pacientes fue de 24 días (con un rango de 9 a 28 días), en contraste con lo reportado en la serie más grande con una mediana de 11 días (rango de 2 a 43 días) ${ }^{5}$. Esto probablemente sea explicable por la complejidad de nuestros pacientes, la necesidad de tratamientos antibióticos prolongados y la limitación a la atención médica ambulatoria en nuestros pacientes.

\section{¿Cómo usar los SPN en infecciones asociadas a dispositivos sin evidencia de endocarditis?}

Se propone el uso inicial de la terapia con los SPN, continúa por 48 horas, con posterior activación de ciclos de 2 minutos de succión y descanso de 3 minutos, con presión inicial de menos de $125 \mathrm{mmHg}$ de presión. Si el paciente se encuentra anticoagulado y no es posible diferir la terapia anticoagulante se propone el uso de la presión negativa continúa con menos de $75 \mathrm{mmHg}^{4,5,10}$. La espuma se debe poner en contacto con el tejido y cerrar el circuito de la presión negativa.

Esta espuma y el sistema debe cambiarse cada 3 días. Según la literatura actual, es razonable realizar un reimplante contralateral al sitio del explante una vez la infección esté controlada ${ }^{4,5,10}$.

\section{¿Cuándo no usar los SPN en infecciones asociadas a dispositivos sin endocarditis?}

Se mencionan como contraindicaciones: la presencia de tejido necrótico, las escaras, la osteomielitis sin tratamiento, las fistulas no tratadas, las heridas por malignidad, la exposición de la vasculatura, los nervios expuestos u órganos expuestos ${ }^{6,10}$. Son ejemplos los pacientes con osteomielitis esternal o clavicular, pacientes con postoperatorios de cirugía ortopédica, pacientes con fistulas crónicas activas de tejido vecino a los bolsillos y pacientes con escoriaciones o úlceras complejas en la piel circundante a sitio de implante.

\section{¿Cuál es la utilidad de la terapia?}

El uso de esta terapia novedosa, incluye procedimientos en pacientes con las IADSE, especialmente, a los que se les realiza capsulectomía completa y extracción manual o que presenten heridas grandes con afrontamiento difícil que requerirían múltiples intervenciones antes de su afrontamiento final.

Es una intervención que ahorra costos.

En nuestra experiencia en el pasado el no uso de los SPN era reemplazado con lavados múltiples, hasta que la herida permitiera el cierre. Esto aumentaba el número de procedimientos posteriores: lavados adicionales, anestesia a repetición, sumándole el dolorcausado al paciente por múltiples intervenciones. El costo aproximado en nuestro medio 
de un procedimiento de extracción manual en donde, además, se implanten los SPN es de \$1'400.000. Resulta ser menor que una extracción complicada que requiere múltiples intervenciones posteriores. Costos evitados por el uso de los SPN.

\section{Conclusiones}

El uso de los sistemas de presión negativa es una antigua herramienta con un uso novedoso en el campo de la electrofisiología.

El uso de los sistemas de presión negativa dentro de un protocolo que incluya: la extracción completa, la capsulectomía, el lavado, la antibioticoterapia sistémica, hace parte del protocolo que proponemos para el manejo agresivo de las IADSE, permitiendo al intervencionista en dispositivos, minimizar las reintervenciones por infección.

Solamente uno de los cinco pacientes descritos requirió reintervención, no obstante, esta fue para extracción de punta del electrodo fracturado y nunca hubo aislamiento microbiológico.

Comparativamente, las series publicadas muestran una mediana de hospitalización menor, pero, con un rango más amplio de los días de hospitalización que lo encontrado en nuestra serie de los pacientes, aunque no se demostró una menor duración de la hospitalización que lo reportado si encontramos ausencia de mortalidad relacionada con SPN y disminución del número de intervenciones posteriores por sangrado del sitio operatorio.

\section{Responsabilidades éticas}

Protección de personas y animales. Los autores declaran que los procedimientos seguidos se conformaron a las normas éticas del comité de experimentación humana responsable y de acuerdo con la Asociación Médica Mundial y la Declaración de Helsinki.

Confidencialidad de los datos. Los autores declaran que han seguido los protocolos de su centro de trabajo sobre la publicación de datos de pacientes.

Derecho a la privacidad y consentimiento informado. Los autores han obtenido el consentimiento informado de los pacientes y/o sujetos referidos en el artículo. Este documento obra en poder del autor de correspondencia.

\section{Conflicto de intereses}

Los autores declaran no tener ningún conflicto de intereses.

\section{Bibliografía}

1. Lee DH, Gracely EJ, Aleem SY, Kutalek SP, Vielemeyer O. Differences of Mortality Rates between Pocket and Nonpocket Cardiovascular Implantable Electronic Device Infections. Pacing Clin Electrophysiol. 2015.

2. Report of a joint Working Party project on behalf of the British Society for Antimicrobial Chemotherapy (BSAC, host organization), British Heart RhSandoe JAT, Barlow G, Chambers JB, Gammage M, Guleri A, Howard P, et al. Guidelines for the diagnosis, prevention and management of implantable cardiac electronic device infection. J Antimicrob Chemother. 2014;70:325-59.

3. Tarakji KG, Chan EJ, Cantillon DJ, Doonan AL, Hu T, Schmitt $\mathrm{S}$, et al. Cardiac implantable electronic device infections: presentation, management, and patient outcomes. Heart Rhythm. Elsevier Inc. 2010;7:1043-7.

4. McGarry TJ, Joshi R, Kawata H, Patel J, Feld G, BirgersdotterGreen UM, et al. Pocket infections of cardiac implantable electronic devices treated by negative pressure wound therapy. Europace. 2014;16:372-7.

5. Shweiki E, Gallagher KE. Negative pressure wound therapy in acute, contaminated wounds: documenting its safety and efficacy to support current global practice. Int Wound J. 2013;10:13-43.

6. Ahsan SY, Saberwal B, Lambiase PD, Koo CY, Lee S, Gopalamurugan $A B$, et al. A simple infection-control protocol to reduce serious cardiac device infections. Europace. 2014;16:1482-9.

7. Huang C, Leavitt T, Bayer LR, Orgill DP. Effect of negative pressure wound therapy on wound healing. Curr Probl Surg. 2014;51:301-31

8. Nof E, Epstein LM. Complications of cardiac implants: Handling device infections. Eur Heart J. 2013;34:229-35.

9. Poller WC, Schwerg M, Melzer C. Therapy of cardiac device pocket infections with vacuum-assisted wound closure-long-term follow-up. PACE - Pacing Clin Electrophysiol. 2012;35:1217-21.

10. The American Association for Thoracic SurgeryPichlmaier $M$, Knigina L, Kutschka I, Bara C, Oswald H, Klein G, et al. Complete removal as a routine treatment for any cardiovascular implantable electronic deviceassociated infection. J Thorac Cardiovasc Surg. 2011;142:1482-90. 